Document downloaded from:

http://hdl.handle.net/10251/148872

This paper must be cited as:

Torregrosa, AJ.; Broatch, A.; Arnau Martínez, FJ.; Hernández-Marco, M. (2017). On the effect of different flux limiters on the performance of an engine gas exchange gas-dynamic model. International Journal of Mechanical Sciences. 133:740-751.

https://doi.org/10.1016/j.ijmecsci.2017.09.029

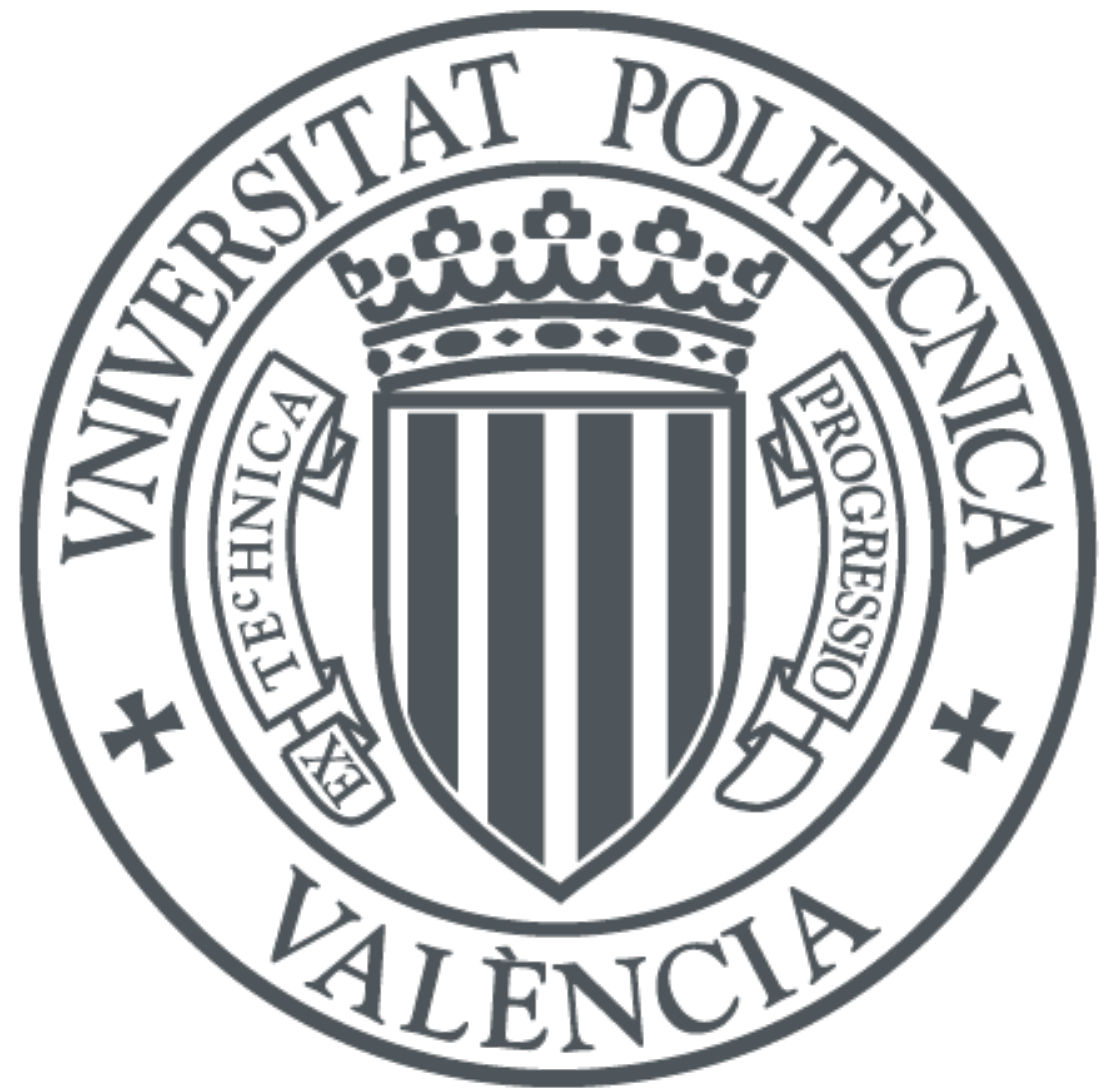

The final publication is available at

https://doi.org/10.1016/j.jmecsci.2017.09.029

Copyright Elsevier

Additional Information 


\title{
On the effect of different flux limiters on the performance of an engine gas exchange gas-dynamic model
}

\author{
A. J. Torregrosa1, A. Broatch, F.J. Arnau, M. Hernández \\ CMT - Motores Térmicos. Universitat Politècnica de València. \\ Aptdo. 22012, E-46071 Valencia, Spain
}

\begin{abstract}
A suitable tool for the design of intake and exhaust systems of internal combustion engines is provided by time-domain non-linear finite volume models. These models, however, are affected by overshoots at discontinuities and numerical dispersion unless some flux limiter is used. In this paper, the effect of the most relevant of such flux limiters on a nonlinear staggered-mesh finite-volume model is evaluated. Flux-Corrected-Transport (FCT) and Total Variation Diminishing (TVD) schemes, together with a Momentum Diffusion Term (MDT) are presented for such a model, and the performance of the resulting methods is checked in different problems representative of the influence of engine gas exchange flows on engine performance and intake and exhaust noise. First, two onedimensional cases are considered: the shock-tube problem, and the propagation of a finite amplitude pressure pulse. Secondly, a simple but representative three-dimensional geometry is studied. From the results obtained, it can be concluded that, even if none of the methods is able to handle properly the three problems considered, the FCT method provides the best overall performance.
\end{abstract}

Keywords: Engine gas exchange, Gas dynamics, Flux limiter

\section{Introduction}

As a consequence of the need of reducing the development time and costs associated with the design of intake and exhaust system for reciprocating internal combustion engines, engine modelling has become an essential engineering tool. Trial-and-error-based design methodologies are still in use, but most of the tests previously performed on prototypes in the early design stages have currently been replaced by numerical computations, and only the most promising solutions are tested in order to fix the final product.

Typically, suitable solutions for modelling both engine performance and intake and exhaust noise have been provided by one-dimensional time domain gas-dynamic codes [1]. The assumption of one-dimensional wave action is legitimate in most of the ducts used in engine intake and exhaust systems, at least in passenger car applications, where duct diameters are relatively small. However, certain elements, most notably duct junctions and intake and exhaust silencers, may exhibit noticeable three-dimensional effects, so that a one-dimensional flow representation would be insufficiently accurate unless a very rough description of such elements were acceptable in the problem under study.

In the case of duct junctions [2] it is the occurrence of complex three-dimensional flow structures what sets the limits for the applicability of simple zero-dimensional

\footnotetext{
${ }^{1}$ Corresponding author: atorreg@mot.upv.es
} 
descriptions [3]. In the case of silencers, the one-dimensional assumption can only be applied to very simple geometries [4] but, even in simple cases such as expansion chambers and Herschel-Quincke tubes, the results are not in general acceptable for frequencies above the cut-on frequency of transversal modes [5]. This represents a serious drawback, especially in the case of the intake system, where underhood packaging necessities give rise to airbox and silencer geometries with low cut-on frequencies, thus impeding a proper assessment even of the low frequency intake noise.

An obvious way to overcome these limitations is the use of computational fluid dynamics (CFD) models; however, the computation time required by their use in a complete intake or exhaust system is excessive. An alternative is provided by using a three-dimensional model only locally, precisely for those elements exhibiting significant three-dimensional effects. This can be achieved by coupling the one-dimensional and three-dimensional models [6], although the proper convergence of the coupling procedure may still imply a considerable computational cost [7].

An attractive possibility arises from the use of Galerkin discontinuous finite element methods, which have been shown to provide good results when applied to onedimensional problems [8], and whose three-dimensional formulation could be used locally in a relatively straightforward manner. However, the computation time is much larger than that required by conventional finite differences techniques, even when suitable simplifications are included [9].

Finally, staggered-mesh finite volume models [10] provide a suitable compromise between the quality of the solution and the computational cost when addressing the prediction of wave dynamics in intake and exhaust systems of internal combustion engines and, in particular, of the effects produced by complex elements. These models have become standard in commercial codes, either as the core of the whole computation [11] [12], or used locally for complex elements exhibiting significant three-dimensional features [13].

It is well known, however, that non-physical oscillations in the flow variables appear when those methods are used in their basic form, most notably at points where significant pressure gradients are present (as usual in the literature, such points will be referred to here as "discontinuities", even if the variations occur over a very small but finite spatial distance). In order to avoid such overshoots at discontinuities, different approaches have been proposed in the literature. The first solutions reported were based on the inclusion in the momentum equation of an additional term, which was an equivalent friction force in the first implementation of the method [14] or, in more recent developments, a momentum diffusion term [15]. An alternative to this solution, suggested by common practice when the flow equations are solved with finite differences schemes, was proposed in [16], where a Flux Corrected Transport (FCT) methodology was used that provided satisfactory results through the combination of dissipation via damping together with the phoenical form of the anti-diffusion term. These two approaches are formally equivalent, as any numerical scheme can be reformulated via Taylor-series expansion as a standard central finite difference scheme with an additional dissipative source term.

However, it has long been known [17] that, under some circumstances, FCT methods can distort the finite-differences solution and produce large errors in mass conservation. Therefore, flux limiters based on the total variation diminishing (TVD) criterion [18] are 
usually preferred in engine gas exchange applications [19], as they allow avoiding such problems and require computational efforts similar to those of the FCT approach [17]. TVD methods are still an active research topic (see e.g. [20]) and have been successfully applied even to extreme flow conditions, such as the propagation of weak shock waves along the exhaust system [21].

The main objective of this paper is to provide a comprehensive evaluation, both in terms of the removal of flow oscillations and of the quality of the frequency domain behaviour of the solution, of the effects of using any of these flux limiters, comprising, as a reference solution, the inclusion of a momentum diffusion term. The structure of the paper is as follows: First, in section 2 the method is described, including the mesh definition and the discretization of the equations and the formulation of the TVD method. Then, in section 3 the different flow limiters and their eventual adaptation to the staggered mesh method is discussed. Subsequently, the shock-tube problem is used in section 4 to test the stability and convergence of the method. In section 5, results of its application to the propagation of a finite amplitude pressure pulse in a duct are shown and discussed by comparison with experimental results in both the time and the frequency domains, while in section 6 a simple geometry exhibiting three-dimensional wave propagation is considered with special emphasis on its frequency domain behaviour. Finally, in section 7 the conclusions of the work are summarized.

\section{Baseline model}

The selected mesh and the basic equations were described by Torregrosa et al. [16], who used a staggered grid consisting of two types of basic elements: volumes and connectors. The former contain information about scalar magnitudes such as pressure, density or temperature, and of course the cell volume itself. The latter contain information on vector quantities (flow velocity or momentum, connector orientation in space), and a scalar quantity (the connector area). It is important to emphasize that a connector always connects two volumes, whereas a volume may be attached to as many connectors as required by the problem considered. In Figure 1, two volumes connected by a connector are shown schematically (volumes do not actually have any defined shape, in the same way as the connector is simply the flow area between the two volumes).

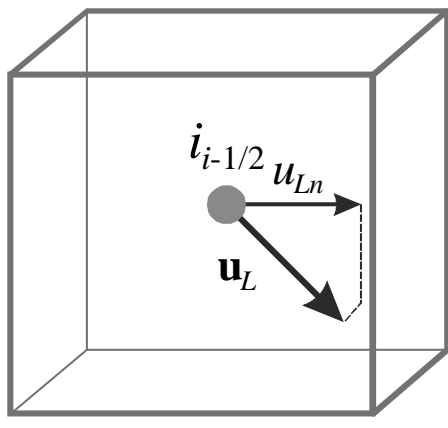

Left volume

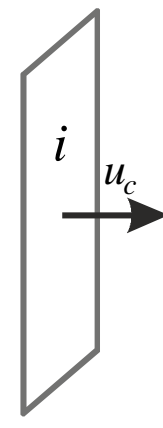

Connector

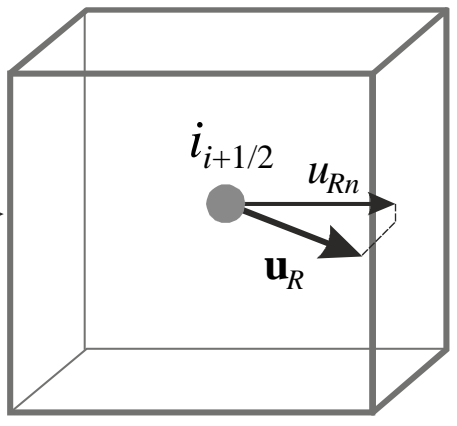

Right volume

Figure 1: Basic mesh elements, definition of velocity projections and notation of volumes and connectors. 
The development of the method starts with the well-known 3D Euler conservation equations without source terms:

$$
\begin{gathered}
\partial \rho / \partial t+\nabla \cdot(\rho \mathbf{u})=0 \\
\partial(\rho \mathbf{u}) / \partial t+\nabla \cdot(\rho \mathbf{u u})=-\nabla p \\
\partial\left(\rho e_{0}\right) / \partial t+\nabla \cdot\left[\left(\rho e_{0}+p\right) \mathbf{u}\right]=0
\end{gathered}
$$

This system of equations is closed with the equation of state of perfect gases.

However, in the current context, the key issue is where and how those equations are solved. The mass equation is solved in the volumes, so that its discretized expression is:

$$
\rho^{n+1}=\rho^{n}+\frac{\Delta t}{V} \sum_{c=1}^{N_{c}} \rho_{c}^{n} u_{c}^{n} A_{c}
$$

where $\rho$ is the density and $u$ is the flow velocity, superscript $n$ indicates the time step, $\Delta t$ represents the time interval, $V$ the volume of the cell, $N_{c}$ the number of connectors and subscript $c$ indicates that the variable is taken at the connectors (otherwise the variable is taken at the volumes).

A similar procedure is then used for the equation of energy, whose resulting discretized expression is:

$$
\left(\rho e_{0}\right)^{n+1}=\left(\rho e_{0}\right)^{n}+\frac{\Delta t}{V} \sum_{c=1}^{N_{c}} \rho_{c}^{n} e_{0}^{n} u_{c}^{n} A_{c}+\frac{\Delta t}{V} \sum_{c=1}^{N_{c}} p_{c}^{n} u_{c}^{n} A_{c}
$$

where $p$ is the pressure and $e_{0}$ is the specific internal energy, which for a perfect gas is:

$$
e_{0}=c_{v} T+u^{2} / 2
$$

The momentum equation is solved at the connectors, and only in the direction orthogonal to the connector surface, by projecting the flow velocity in the connected volumes onto that direction, as depicted in Figure 1 , where the velocity $u_{c}$ in the connector, and the projections of the volume flow velocity, $u_{L n}$ and $u_{R n}$, are shown. Based on this assumption, it follows that one can compute the momentum in the connector by solving a onedimensional momentum equation, whose discretization along the same lines as in the previous cases gives:

$$
\left(\rho_{c} u_{c} A_{c}\right)^{n+1}=\left(\rho_{c} u_{c} A_{c}\right)^{n}+(\Delta t / \Delta L)\left[\left(\rho u_{n}^{2}+p\right)_{L}+\left(\rho u_{n}^{2}+p\right)_{R}\right] A_{c}
$$

Here, $u_{n}$ denotes the velocity projection onto the direction orthogonal to the connector surface and subscripts $R$ and $L$ refer to the volumes at the right and left of the connector, respectively. It is worth noticing that, with this simplification, a one-dimensional equation for each connector must be solved, instead of three coupled equations for each volume, which significantly reduces the computation time. This quantity is then used in the mass and energy conservation equations for the next time step. In the case of the energy and mass equations, some additional scalar terms from the connectors, such as density or pressure, are needed. These values are calculated by an upwind approach, so that they are taken from the right or left volumes, depending on the flow direction.

Finally, the momentum associated with the volumes is calculated by distributing the connector momentum between the two adjacent volumes according to their relative sizes. 
In uniform meshes, half the momentum of the connector is thus assigned to each volume. As the orientation of the connectors is also known, the momentum vector of each volume is obtained from the vector sum:

$$
\left(\rho_{c} \mathbf{u} V\right)_{v}^{n+1}=\frac{1}{2} \sum_{c=1}^{N_{c}}\left(\rho \mathbf{u}_{c} A_{c} \Delta L\right)_{c}^{n+1}
$$

With the previous prescription, the method turns out to be a second-order accuracy method based on an explicit scheme with a staggered grid, as shown in Figure 2.

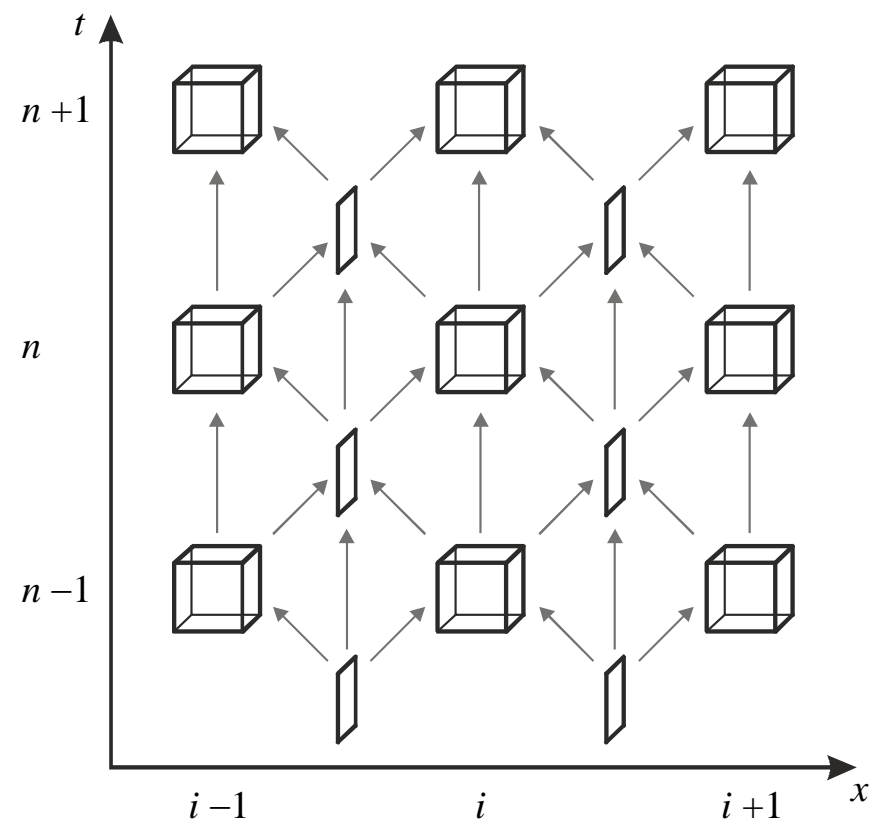

Figure 2: Scheme of the staggered mesh and the associated time marching.

\section{Flux limiters}

As pointed out in section 1, the method described above does not satisfy the stability requirement, since nonphysical oscillations may appear, especially in cases where pressure gradients are significant. This is a very common situation when simulating flows associated with engine gas exchange due to their pulsating nature. Since simulations with unsteady mean flow or high amplitude pressure perturbations are one of the main goals of the method, a stabilization technique must be developed and applied to avoid spurious oscillations. Most of the stabilization methods available in the literature are based on the correction of the flux terms present in the equations, and have been developed for finite differences schemes, such as the Lax-Wendroff method [22]. In the method used here, the solution of the momentum equation in the connectors is used to compute the fluxes required for the mass and energy equations in the volumes, whence the stabilization method should only be applied to the momentum equation. This fact will be taken into account when adapting a stabilization method previously developed for a finite difference method scheme.

In this section, the formulation of the three different flux limiters considered is described. In the case of the FCT and the TVD techniques, methods developed for finite difference 
schemes are adapted so that they can be used in conjunction with the staggered grid finitevolume method described above.

\subsection{Momentum diffusion term}

The basic concept is adding a diffusion term to the momentum equation so that the mass flux computed at the corresponding connector is conveniently limited. With this purpose, the momentum flux density tensor used in the momentum equation (2) is modified, in a way similar to that used for incorporating viscosity effects, as follows:

$$
\partial(\rho \mathbf{u}) / \partial t+\nabla \cdot(\rho \mathbf{u u}+\mathbf{D})=-\nabla p
$$

where tensor $\mathbf{D}$ is assumed to depend linearly on the local momentum gradients, i.e.:

$$
\mathbf{D}=\epsilon \nabla(\rho \mathbf{u})
$$

Here, the scalar quantity $\epsilon$ has the dimensions of a kinematic viscosity and can thus be interpreted as a momentum diffusion coefficient. With this prescription, the contribution of the diffusion term $\nabla \cdot \mathbf{D}$ will only be relevant if significant gradients exist, and any resulting spurious oscillations will be damped.

Projection of equation (10) onto the direction of a connector and subsequent discretization in the same way as for equation (7) gives

$$
\left(\rho_{c} u_{c} A_{c}\right)^{n+1}=\left(\rho_{c} u_{c} A_{c}\right)^{n}+\left(\frac{\Delta t}{\Delta L}\right)\left\{\left[\left(\rho u_{n}^{2}+p\right)_{L}+\left(\rho u_{n}^{2}+p\right)_{R}\right] A_{c}+\left(\widetilde{D}_{L n}-\widetilde{D}_{R n}\right)\right\}
$$

where $\widetilde{D}_{L}$ and $\widetilde{D}_{R}$ are the projections onto the connector direction of tensor $\widetilde{\mathbf{D}}=\epsilon \nabla(\rho \mathbf{u} A)$ computed in the two adjacent volumes. Following [15], the momentum diffusion coefficient is evaluated by considering the mesh size and the time step in relation with the local flow velocity at the volume, as

$$
\epsilon=\frac{|\mathbf{u}|}{2}(\Delta L-|\mathbf{u}| \Delta t)
$$

and the gradient of mass flow rate $\nabla(\rho \mathbf{u} A)$ is computed from the projections of the mass flow rates of the adjacent connectors onto each direction.

\subsection{Flux-Corrected-Transport formulation and scheme}

When applied to a finite difference scheme, FCT consists of three stages [23]: a transport stage based on the scheme considered, a diffusion stage for reducing the numerical dispersion introduced in the transport stage, and an anti-diffusion stage to restore the accuracy of the scheme at cells with a smooth solution while preserving the diffusion operator accuracy in the vicinity of discontinuities. The diffusion operator is defined as:

$$
D_{i}(W)=\theta\left(W_{i+1 / 2}\right)-\theta\left(W_{i-1 / 2}\right)
$$

with

$$
\theta\left(W_{i+1 / 2}\right)=\left(W_{i+1}-W_{i}\right) \vartheta / 4
$$

Here, $W_{i}$ is the variable computed at cell $i$ in the transport stage, and subscripts $i \pm 1 / 2$ indicate that the variable is evaluated at the midpoint between cells $i$ and $i \pm 1$. The factor $\vartheta$ is a positive real number taken to be $\vartheta \geq 1 / 2$ so that instabilities are avoided. According to [16] it is preferable to apply diffusion via damping in order to compute the guessed value $\bar{W}_{i}$ of variable $W_{i}$, so that: 


$$
\bar{W}_{i}^{n+1}=W_{i}^{n+1}+D_{i}\left(W^{n}\right)
$$

For the case of a staggered-mesh finite-volume method, since a connector always connects two volumes, the obvious adaptation procedure would be to consider that the FCT cells correspond to the connectors, and to use the projection of the variables corresponding to the volumes connected by connector $i$ at the intermediate steps $i \pm 1 / 2$. Then, the momentum equation with diffusion via damping would be as follows:

$$
\left(\overline{\rho u_{c} A_{c}}\right)_{i}^{n+1}=\left(\rho u_{c} A_{c}\right)_{i}^{n+1}+D_{i}\left(\rho u_{c} A_{c}\right)_{i}^{n}
$$

and thus the diffusive term becomes:

$$
D_{i}\left(\rho u_{c} A_{c}\right)_{i}^{n}=\theta\left(\rho u_{n} A_{c}\right)_{i+1 / 2}^{n}-\theta\left(\rho u_{n} A_{c}\right)_{i-1 / 2}^{n}
$$

where

$$
\theta\left(\rho u_{c} A_{c}\right)_{i+1 / 2}^{n}=\left[\left(\rho u_{n} A_{c}\right)_{i+1}^{n}-\left(\rho u_{n} A_{c}\right)_{i}^{n}\right] \vartheta / 4
$$

Finally, the non-linear anti-diffusion operator $A_{i}$ is defined as:

$$
A_{i}(W)=\Psi\left(W_{i-1 / 2}\right)-\Psi\left(W_{i+1 / 2}\right)
$$

Making use of the anti-diffusive limited flow defined in [24] gives:

$$
\Psi\left(W_{i+1 / 2}\right)=s \max \left[0, \min \left((5 / 8) s \Delta W_{i-1 / 2},(1 / 8)\left|\Delta W_{i+1 / 2}\right|,(5 / 8) s \Delta W_{i+3 / 2}\right)\right]
$$

Here, $\quad \mathrm{s}=\operatorname{sign}\left(\Delta W_{i+1 / 2}\right), \Delta W_{i-1 / 2}=W_{i}-W_{i-1}, \Delta W_{i+1 / 2}=W_{i+1}-W_{i} \quad$ and $\quad \Delta W_{i+3 / 2}=$ $W_{i+2}-W_{i+1}$. Then, according to [16], the phoenical form should be used, so that:

$$
\bar{W}_{i}^{n+1}=\bar{W}_{i}^{n+1}+A_{i}\left(W^{n+1}\right)
$$

As they have been defined, flux correction techniques are conservative at the interior mesh points, since all the corrections are cancelled out along the duct except at the ends. In this last instance, the anti-diffusion operator can be defined by evaluating the differences present in each case, i.e.:

$$
\Psi\left(W_{i+1 / 2}\right)=s \max \left[0, \min \left((5 / 8) s \Delta W_{i-1 / 2},(1 / 8)\left|\Delta W_{i+1 / 2}\right|,\right)\right]
$$

at the right end, and

$$
\Psi\left(W_{i-1 / 2}\right)=s \max \left[0, \min \left((1 / 8)\left|\Delta W_{i-1 / 2}\right|,(5 / 8) s \Delta W_{i+1 / 2},\right)\right]
$$

at the left end.

Equations (19) to (23) for the anti-diffusion stage can be adapted to the staggered-mesh finite-volume method in a way similar to that used for the diffusion stage.

\subsection{Total-Variation-Diminishing formulation and scheme}

Again, first the formulation of the method for finite differences schemes will be outlined, and then its adaptation to a staggered-mesh method will be described.

Following Harten [18], consider an explicit finite difference scheme in conservation form, denoted in operator form as

$$
w^{n+1}=L \cdot w^{n}
$$

Then, the finite difference scheme is total variation diminishing (TVD) if for all $w$ with bounded total variation one has 


$$
T V\left(w^{n+1}\right)=T V\left(L \cdot w^{n}\right) \leq T V\left(w^{n}\right)
$$

where the total variation is defined, for a discrete function $w$, as

$$
T V(w)=\sup \sum_{i}\left|w_{i+1}-w_{i}\right|
$$

Harten [18] proved that TVD schemes are intrinsically free from spurious oscillations.

Several TVD schemes have been developed for finite difference schemes, most notably by Harten [18], Sweby [25] and Davis [26]. A comparison of these methods was performed by Arnau [27] on a finite differences scheme, showing that all of them brought in a noticeable improvement with respect to the Lax-Wendroff scheme, most notably when the schemes proposed by Davis and Harten were used. However, while the Davis TVD flux limiter needed only about twice the time than the original Lax-Wendroff scheme, the Harten TVD flux correction method increased the computation time by around fifteen times the original, due to the computation of the Jacobian matrix. The Davis TVD flux limiter method was thus selected for its adaptation to the staggered grid.

The method proposed by Davis [26] results in the addition of the term

$$
\left[\bar{G}^{+}\left(r_{i}^{+}\right)+\bar{G}^{-}\left(r_{i+1}^{-}\right)\right] \Delta \boldsymbol{W}_{i+1 / 2}^{n}-\left[\bar{G}^{+}\left(r_{i-1}^{+}\right)+\bar{G}^{-}\left(r_{i}^{-}\right)\right] \Delta \boldsymbol{W}_{i-1 / 2}^{n}
$$

to the second step of either a two-step Lax-Wendroff scheme or of a MacCormack scheme. The function $\bar{G}^{ \pm}$in equation (27) is defined as

$$
\bar{G}^{ \pm}\left(r_{i}^{ \pm}\right)=\xi C(v)\left[1-\psi\left(r_{i}^{ \pm}\right)\right]
$$

where $\xi=0.5$ and $C(v)$ is a function of the Courant number given by

$$
C(v)= \begin{cases}v(1-v) & v \leq 0.5 \\ 0.25 & v>0.5\end{cases}
$$

and finally

$$
r_{i}^{+}=\frac{\left[\Delta \boldsymbol{W}_{i-1 / 2}^{n}, \Delta \boldsymbol{W}_{i+1 / 2}^{n}\right]}{\left[\Delta \boldsymbol{W}_{i+1 / 2}^{n}, \Delta \boldsymbol{W}_{i+1 / 2}^{n}\right]}, \quad r_{i}^{-}=\frac{\left[\Delta \boldsymbol{W}_{i-1 / 2}^{n}, \Delta \boldsymbol{W}_{i+1 / 2}^{n}\right]}{\left[\Delta \boldsymbol{W}_{i-1 / 2}^{n}, \Delta \boldsymbol{W}_{i-1 / 2}^{n}\right]}
$$

Here, [ . . . ] denotes the inner product of two vectors, and $\Delta \boldsymbol{W}_{i+1 / 2}^{n}=\boldsymbol{W}_{i+1 / 2}^{n}-\Delta \boldsymbol{W}_{i}^{n}$.

Regarding the limiter used in equation (28), Davis proposed the following expression

$$
\psi(r)=\min (2|r|, 1)
$$

which admits Courant numbers of up to 0.95 .

As in the two previous cases, the flux limiter should only be added to the momentum equation, using the required variables from the neighbour connectors. The momentum in the connectors is then modified by adding the term:

$$
(\bar{W})^{n+1}=(W)^{n+1}+\left[\bar{G}^{+}\left(r_{i}^{+}\right)+\bar{G}^{-}\left(r_{i+1}^{-}\right)\right] \Delta W_{i+1 / 2}^{n}-\left[\bar{G}^{+}\left(r_{i-1}^{+}\right)+\bar{G}^{-}\left(r_{i}^{-}\right)\right] \Delta W_{i-1 / 2}^{n}
$$

where $W=\rho_{c} u_{c} A_{c}$ is the momentum calculated with equation (7). The rest of the terms are calculated as described by Davis in equations (28) to (31), most notably the $r^{ \pm}$ expressions (30), where there is an inner product of two vectors formed by the conservative variables: 


$$
\Delta \boldsymbol{W}_{i+1 / 2}^{n}=\left[\begin{array}{c}
\Delta \rho_{c}^{n} \\
\Delta\left(\rho_{c} u_{c} A_{c}\right)^{n} \\
\Delta\left(\rho_{c} e_{0, c}\right)^{n}
\end{array}\right]
$$

Despite the mass and energy equations are evaluated at the volumes, their values for the $\Delta \boldsymbol{W}_{i+1 / 2}^{n}$ vector must be taken from the connectors for consistency, for which again an upwind approach will be adopted.

The only issue when trying to adapt the Davis flux limiter method to a staggered grid is related with end-volumes. As seen in equation (30), conservative variables of neighbour connectors from both sides are needed to compute the flux limiter in each connector. Furthermore, equation (27) uses the terms $r_{i+1}^{-}$and $r_{i-1}^{+}$, which are computed from the conservative variables of two neighbour connectors on each side. This is not a serious problem in a one-dimensional model, since only two cells of each side of the modelled duct will be affected, and the problem can be solved by assuming that the values of the variables corresponding to the non-existent neighbour cells are the same as those of the end-cell. This approximation has been used in one-dimensional models with good results. However, when modelling a more complex geometry with a three-dimensional mesh, many end-volumes may appear and the effect of the simplifications adopted can be much more significant.

In the method developed, the solution adopted was using the value of the conservative variables of the end connector, but inverting the sign of the momentum so that the resultant momentum at the wall is zero. This approximation has given good results when applied to meshes with a sufficiently large number of cells in each direction, although the solution tends to be more diffusive.

\section{Application to the shock-tube problem}

As a first validation of the method capabilities, it is commonplace to consider the shocktube problem [28]. In this problem, two gases with different thermo- and fluid-dynamic states are put into contact. In Figure 3(a) an outline of the initial state of the problem is shown. As time progresses, the contact discontinuity travels with the flow velocity, whereas a shock wave propagates in the same direction at a velocity corresponding to the addition of the speed of sound and the flow velocity, and a rarefaction wave whose propagation velocity is the speed of sound minus the flow velocity travels in the opposite direction. These perturbations define four zones with different thermo- and fluid-dynamic states, as shown in Figure 3(b). Since all kinds of possible propagating perturbations (contact discontinuity, and shock and rarefaction waves) are present and easy to identify in the solution, this problem is often used in the literature. 
(a)

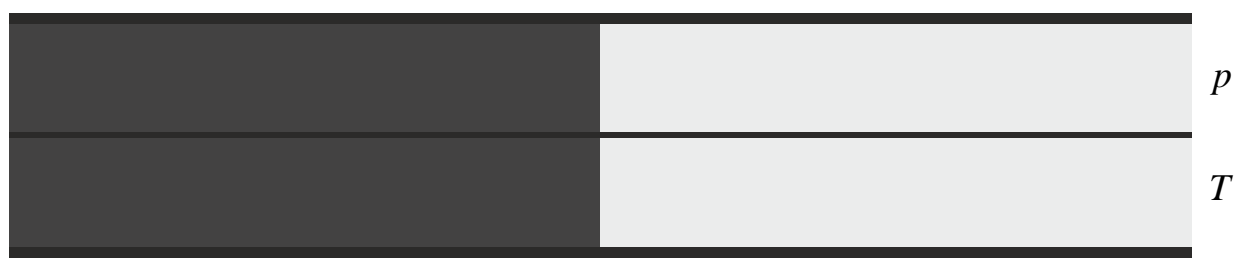

(b)

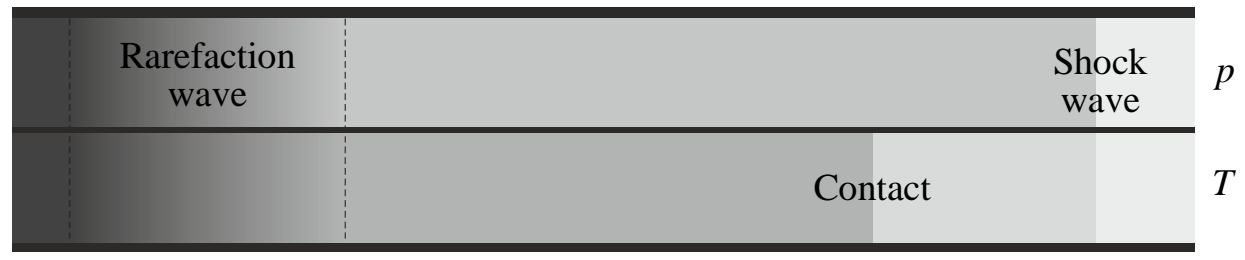

Figure 3: Initial state of the shock-tube problem (a) and scheme of the solution structure after a certain time (b).

It is worth mentioning that, as in any other method of this nature, the CFL condition needs to be satisfied. This condition, obtained by Courant, Friedrichs and Lewy [29] in the context of the demonstration of the existence of a solution to certain partial differential equations (PDE), establishes that, for any numerical method, the domain of dependence of the method should include the domain of dependence of the PDE, at least in the limit when the mesh size $\Delta x$ and the time step $\Delta t$ tend to 0 . This condition is usually expressed in term of the Courant number $v$ as:

$$
v=\lambda \Delta t / \Delta x<1
$$

where $\lambda$ is the propagation speed of the signal, which typically corresponds to the addition of the speed of sound and the flow velocity. In the case of the staggered-mesh finite volume model presented, the CFL condition can be expressed as:

$$
v=\lambda \Delta t A_{c} / V<1
$$

After securing the CFL condition, the staggered-mesh finite-volume method was applied to the shock-tube problem. The initial conditions chosen were: $p_{1}=3.5 \mathrm{bar}, p_{4}=0.5 \mathrm{bar}$, $T_{1}=2800 \mathrm{~K}, T_{4}=300 \mathrm{~K}$, and $u_{1}=u_{4}=0 \mathrm{~ms}^{-1}$. A $1 \mathrm{D}$ mesh was used, with 250 volumes 4 mm long.

In Fig. 4 comparison is given between the analytical solution provided in [28] and the results obtained with the raw method without any flux limiter and with those obtained by including the momentum diffusion term. A noticeable overshooting associated with the propagation of the shock wave can be clearly seen in the basic solution, whereas it can be observed that those overshoots have been successfully removed by the inclusion of the momentum diffusion term. This appears to be the only point in which, as expected, this procedure produces significant changes in the solution, as the other features worth noticing are present in the two solutions shown: the smoothing effect on the trailing side of the leftwards-moving rarefaction wave, which is specially clear in the pressure and velocity plots, and a certain deviation from the analytical solution around $x=0.65 \mathrm{~m}$ in the pressure and velocity plots. Such deviation, that appears as an overestimation of the pressure value and an underestimation of the velocity magnitude, occurs precisely at the position of the contact discontinuity in the analytical solution for density and temperature (the deviations observed in the density and the temperature will be commented later). 

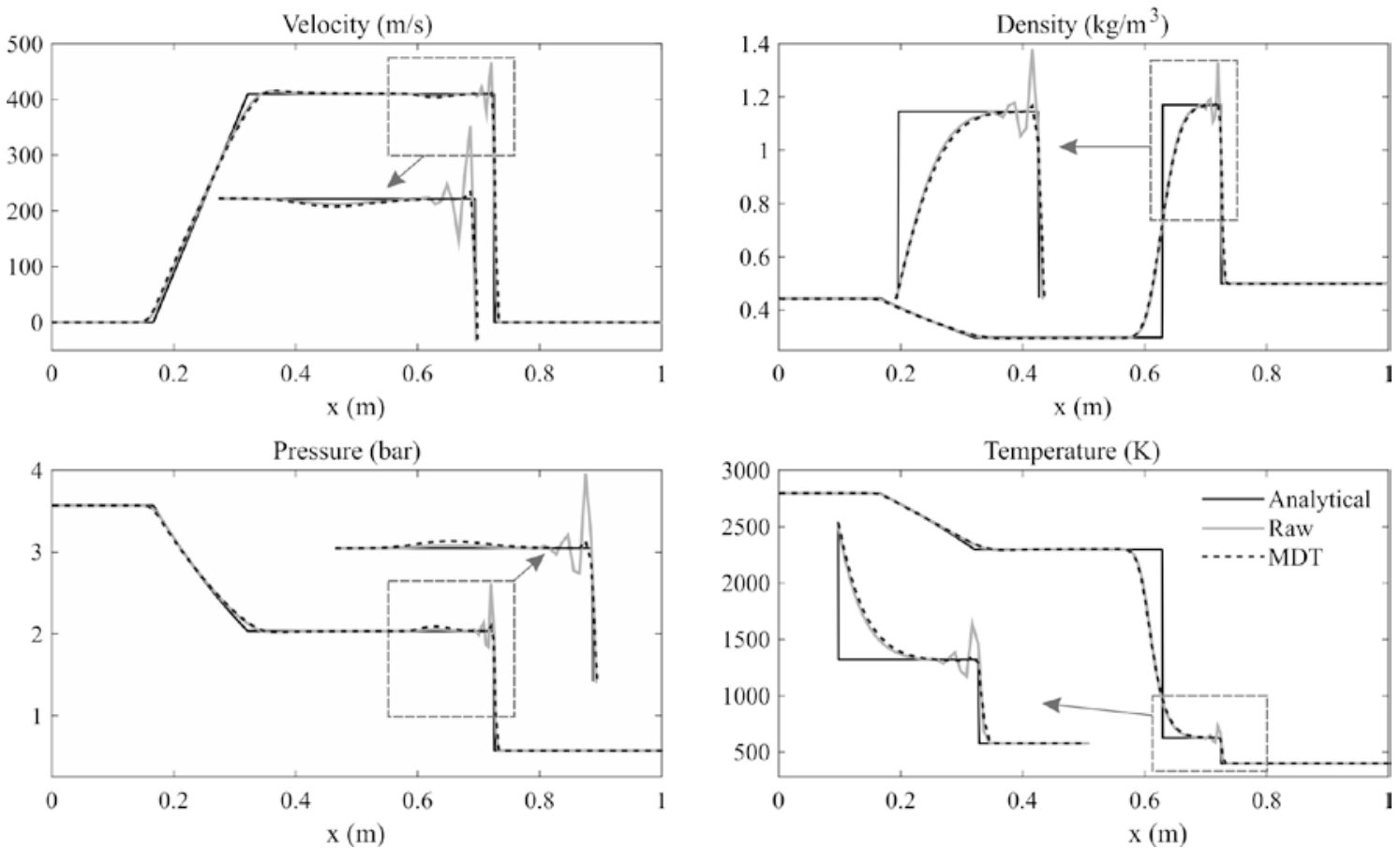

Figure 4: Comparison of solutions of the shock-tube problem: analytical, obtained with the original (raw) method and with the momentum diffusion term (MDT).

In Fig. 5, the same representation is given for the results obtained with the FCT method proposed in [16] and the TVD scheme presented here. Again it can be observed that any overshoots have been suitably removed by the FCT method and, to a lesser extent, by the inclusion of the additional TVD terms, and that in both cases the smoothing effect on the trailing side of the leftwards-moving rarefaction wave is apparent.
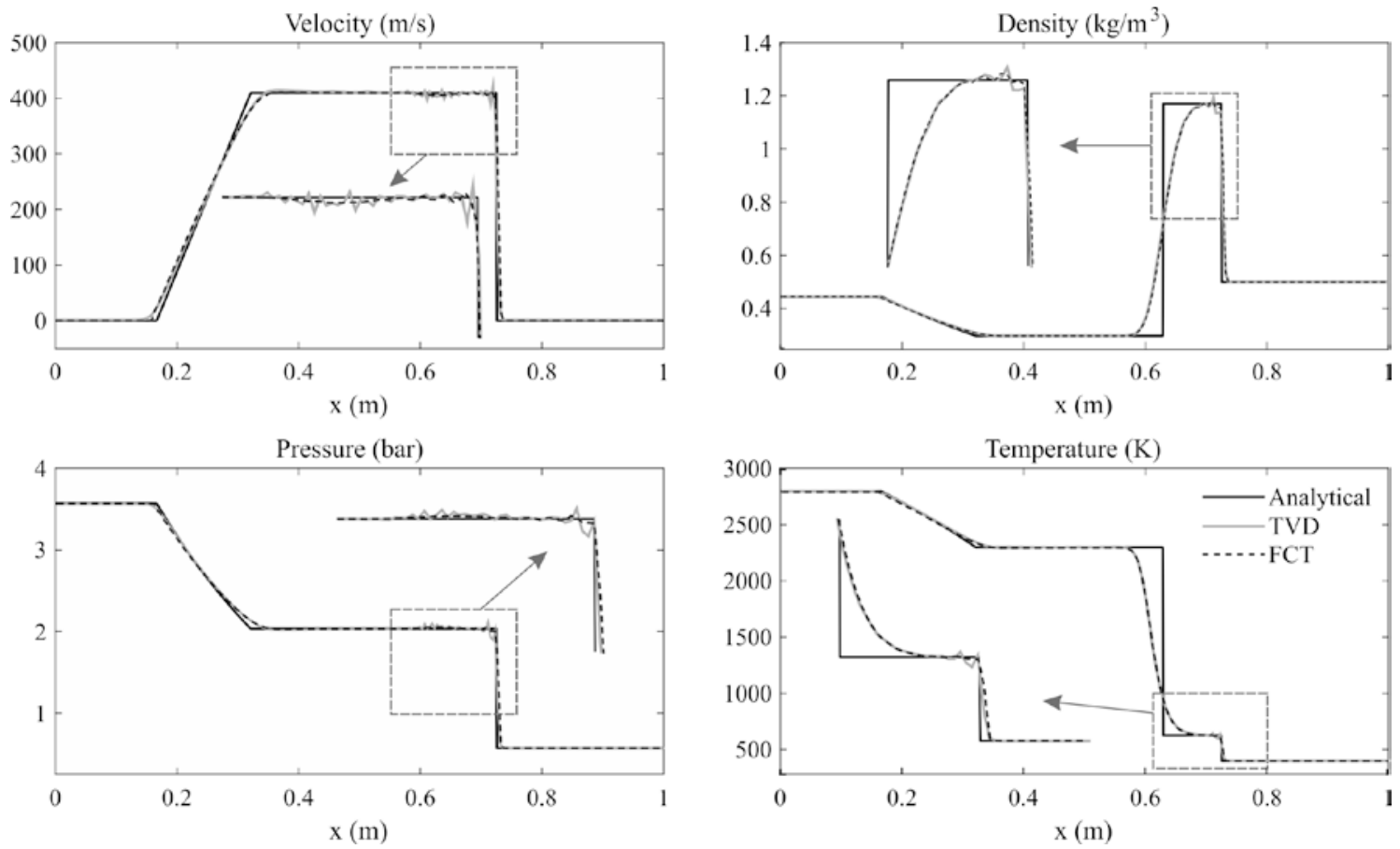

Figure 5: Comparison of solutions of the shock-tube problem: analytical, obtained with the TVD method and the FCT method. 
Regarding the deviation noticed above at the theoretical position of the contact discontinuity, it can be observed that, regardless of the correction method used, the contact discontinuity spreads in space, and thus the methods are introducing some diffusion at this point due to the procedure used for the solution of the mass and energy equations. When this information is fed back to the momentum equation the deviations observed in pressure and velocity are produced, as the flat profiles observed in the analytical solution are only compatible with a real discontinuity. Additionally, it can be observed that a small perturbation appears in the TVD solution for pressure and velocity that might indicate that, even if the density and the temperature seem to be correctly computed, the effect of the spread contact discontinuity on the momentum equation has not been properly handled.

While the spreading of the contact discontinuity could be considered as a serious shortcoming for the description of actual shock waves, this will not in general be the case in practical engine applications, as actual discontinuities occurring in those flow situations are much less abrupt. Additionally, one should expect that this issue should be strongly dependent on the discretization used, and this is confirmed by Fig. 6, where density and pressure results obtained by using 250, 500 and 1000 volumes with the three correction methods considered are shown. As expected, the results for both magnitudes are closer to the analytical solution as the number of volumes increases. It is also worth mentioning that the perturbation observed in the pressure obtained with the TVD method is reduced, even if it has not totally disappeared, while the rest of the methods exhibit a smooth behaviour that even improves when increasing the number of volumes.
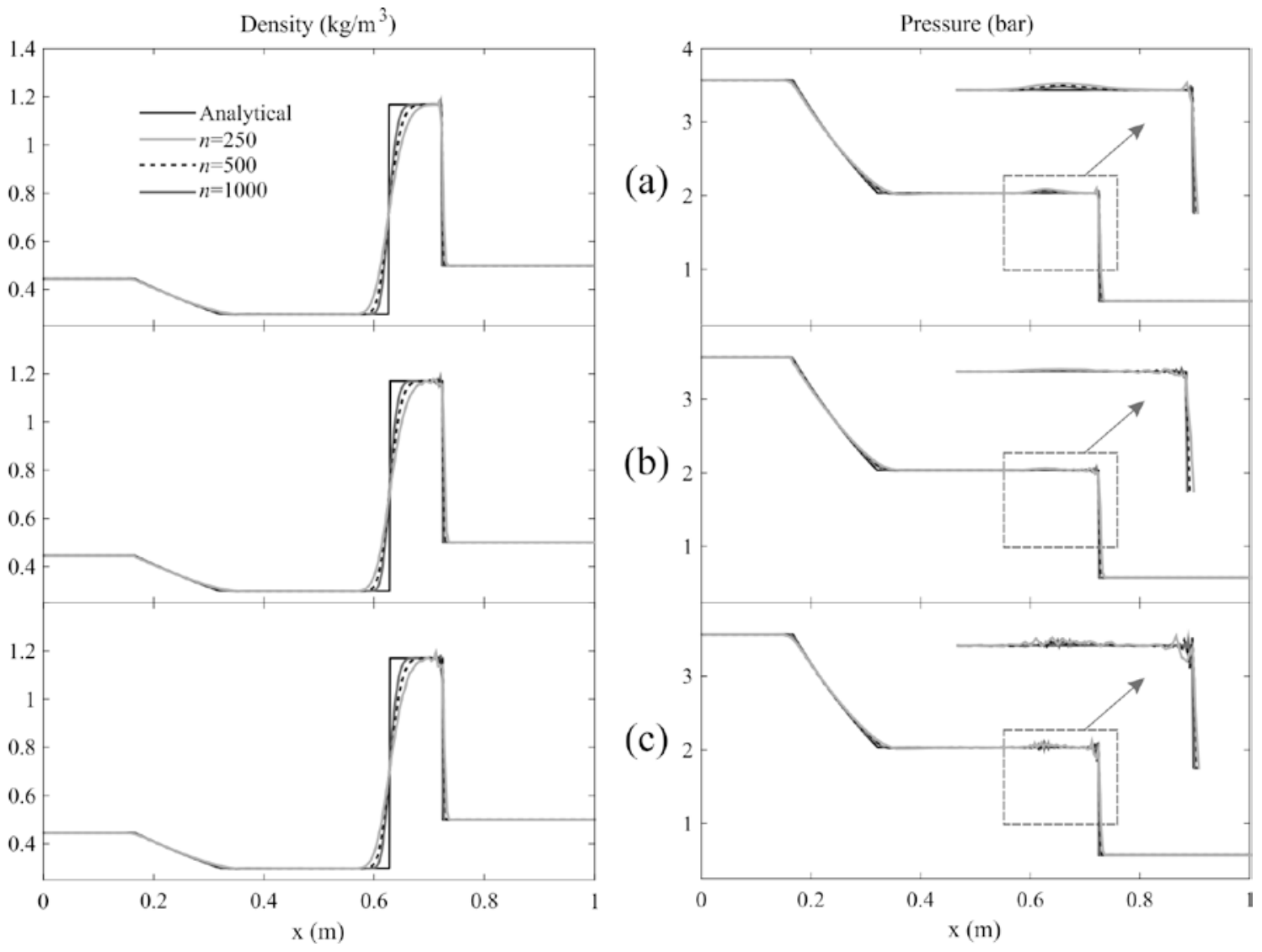

Figure 6: Effect of the discretization used on the description of the contact discontinuity for the three flux limiters considered: (a) MDT, (b) FCT, (c) TVD. 
In order to quantify the previous considerations, the $L^{2}$ and $L^{\infty}$ norms of the deviation of all the variables corresponding to the cases represented in Fig. 6 were computed. These norms are defined as

$$
\begin{aligned}
L^{2} & =\left(\sum_{i=1}^{N_{v}} x_{i}^{2}\right)^{1 / 2} \\
L^{\infty} & =\max \left(\left|x_{i}\right|\right)
\end{aligned}
$$

where $x_{i}$ is the deviation between the analytical solution and the model prediction in each volume or connector, depending on the variable to which it is applied. These two norms were chosen because the $L^{2}$ norm provides a mean global assessment of the deviation obtained, which is complemented by the local view provided by the $L^{\infty}$ norm.

The results are shown in Table 1 where, in order to make the results comparable, the $L^{2}$ norm was divided by the number of volumes $N_{v}$ used in each case. Consistently with the results shown in Fig. 6 , the results for $L^{2}$ improve when increasing $N_{v}$ for the three methods. This is not the case, however, for $L^{\infty}$, with a rather erratic behaviour and tendencies that change with the magnitude and the method considered. Therefore, increasing $N_{v}$ produces an overall improvement in the solution, but significant local differences may still occur.

Considering now the comparison between the three methods, it can be observed that, for a sufficiently high number of volumes $\left(N_{v}=1000\right)$, the performance of the three methods is comparable, except in the case of the velocity, in which the momentum diffusion term provides significantly lower values for both norms. In the intermediate case of $N_{v}=500$ the results are again comparable both in terms of $L^{2}$ and $L^{\infty}$, as none of the methods provides the lowest value for all the magnitudes: the best result for pressure is given by the FCT technique whereas the best result for velocity is that obtained with the MDT method, but all of them within the same order of magnitude. This situation is somehow reversed in the case $N_{v}=250$, for which the best result for pressure is given by the MDT

\begin{tabular}{|c|c|c|c|c|c|c|c|c|c|c|}
\hline \multirow[t]{2}{*}{$N_{v}$} & & \multicolumn{3}{|c|}{250} & \multicolumn{3}{|c|}{500} & \multicolumn{3}{|c|}{1000} \\
\hline & & FCT & TVD & MDT & FCT & TVD & MDT & FCT & TVD & MDT \\
\hline \multirow{4}{*}{$L^{2} / N_{v}$} & $u$ & 0.9949 & 1.6172 & 1.502 & 0.4839 & 0.50147 & 0.4399 & 0.3151 & 0.3038 & 0.267 \\
\hline & $\rho$ & 0.0043 & 0.0042 & 0.00415 & 0.0022 & 0.00227 & 0.0023 & 0.0014 & 0.0014 & 0.0014 \\
\hline & $p$ & 0.0038 & 0.0026 & 0.0023 & 0.0007 & 0.0008 & 0.00093 & 0.0007 & 0.0006 & 0.0005 \\
\hline & $T$ & 21.499 & 21.698 & 21.823 & 14.621 & 14.651 & 14.719 & 9.8141 & 9.845 & 9.88 \\
\hline \multirow{4}{*}{$L^{\infty}$} & $u$ & 220.49 & 363.01 & 342.85 & 223.56 & 231.27 & 196.51 & 236.59 & 213.26 & 181.22 \\
\hline & $\rho$ & 0.4218 & 0.4092 & 0.4158 & 0.4056 & 0.4146 & 0.416 & 0.4131 & 0.4217 & 0.424 \\
\hline & $p$ & 0.8965 & 0.5422 & 0.4322 & 0.1408 & 0.1922 & 0.3097 & 0.6596 & 0.4812 & 0.364 \\
\hline & $T$ & 1566.1 & 1573.2 & 1581.1 & 1588.4 & 1578.9 & 1590.3 & 1601.1 & 1600.9 & 1606.7 \\
\hline
\end{tabular}
technique whereas the best result for velocity is that obtained with the FCT method.

Table 1: Comparison of the $L^{2}$ and $L^{\infty}$ norms of the deviation between the analytical shocktube solution and the different correction methods used. 


\section{Experimental assessment}

While the shock-tube problem provides a comprehensive view of the performance of the different schemes in terms of the spatial distribution (or, equivalently, of the time evolution at a given point) of the resulting flow perturbations, it is not well suited for studying the behaviour of the methods in the frequency domain. This results from the fact that, for a given location, the phenomenon studied takes places in a very short time interval (less than $1 \mathrm{~ms}$ ), so that accounting for the details of the different solutions implies a very poor frequency discretization and, consequently, an insufficient amount of information in the frequency range of interest for engine gas exchange studies.

Therefore, a different approach was chosen in order to study the frequency domain behaviour of the different schemes. This consisted in the consideration of the propagation in a straight duct of a finite amplitude pressure pulse whose characteristics (amplitude and duration) were chosen so as to guarantee a sufficient frequency resolution in the frequency range of interest as well as a sufficient excitation level at all the relevant frequencies, i.e. a substantially flat spectrum [30]. The experimental setup is shown in Fig. 7 together with an approximate representation of the pulse propagation in the $(x, t)$ plane.

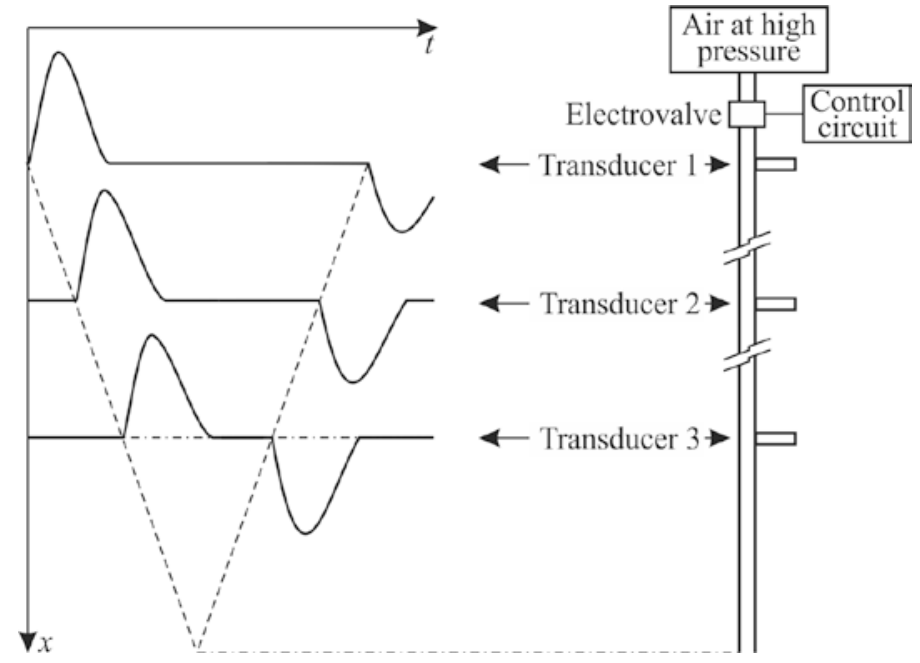

Figure 7: Experimental set-up and representation of pressure pulse propagation.

The pulse was generated through the controlled discharge from a high-pressure tank into the duct, making use of a fast-operation electrovalve [31]. Then, the resulting pulse was recorded $15 \mathrm{~m}$ downstream of the valve (transducer 1 in Fig. 7), at a second station $10 \mathrm{~m}$ downstream of the first one (transducer 3 ) and at a third station $10 \mathrm{~m}$ downstream of the second one and separated $15 \mathrm{~m}$ from the downstream open end (transducer 3 ). In this way, proper development of the pulse into a weakly nonlinear perturbation is allowed, and it is possible to avoid any overlap with the pulse reflected by the open end, as indicated in Fig. 7. The pulse recorded by transducer 1 and its frequency spectrum are shown in Fig. 8, where it can be observed that actually the pulse at this position is not yet fully developed, as indicated by the features present in the spectrum.

The time domain pressure record was introduced as a boundary condition to the different methods considered. As this is an essentially forward-moving perturbation, it is well suited for a representation in terms of the Riemann variables of the method of 
characteristics, and thus the boundary condition was formulated accordingly, along the lines indicated in [32].
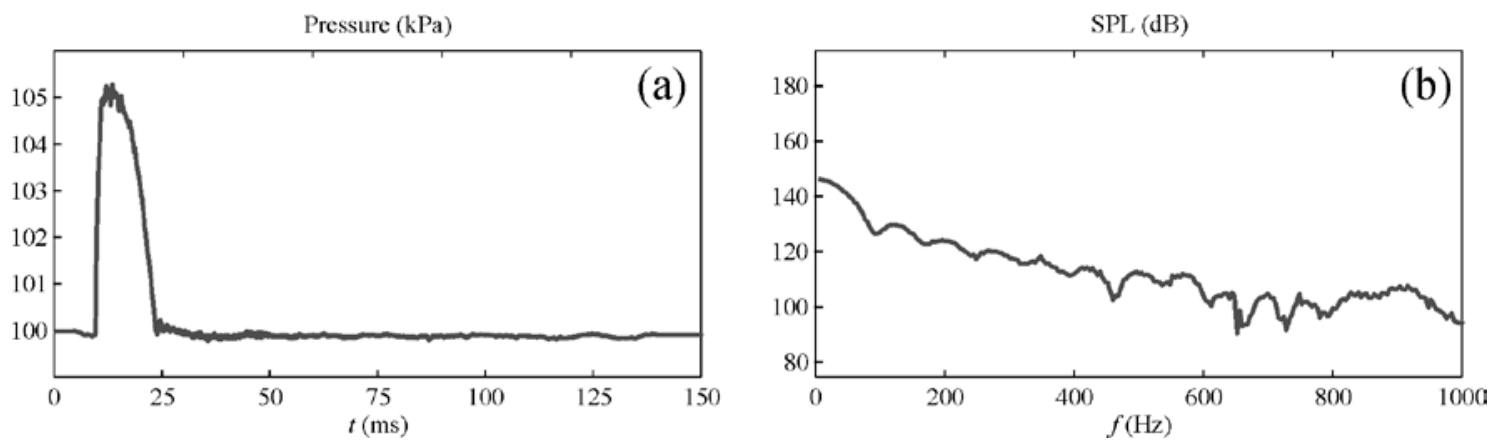

Figure 8: Pressure pulse recorded at first measurement station, and later used as boundary condition for the computations: (a) time domain, (b) frequency domain.

The results obtained at the two other measurement stations are shown in Fig. 9 for the different flux limiters considered (the results of the raw method exhibited very large oscillations and have been omitted for clarity). It is apparent in the time domain representation of Fig 9(a) that any overshoots associated with the original method appear to be substantially removed by the three methods, except in the vicinity of the rising ramp of the pulse. In this case, only the TVD method is able to damp the pressure oscillations and produce a result closer to the measurement. Apart from this, all the methods reproduce the overall shape of the pulse, except in the decay zone, where differences between the different methods are negligible, but none of them accounts for the gradual decay observed in the measurement.
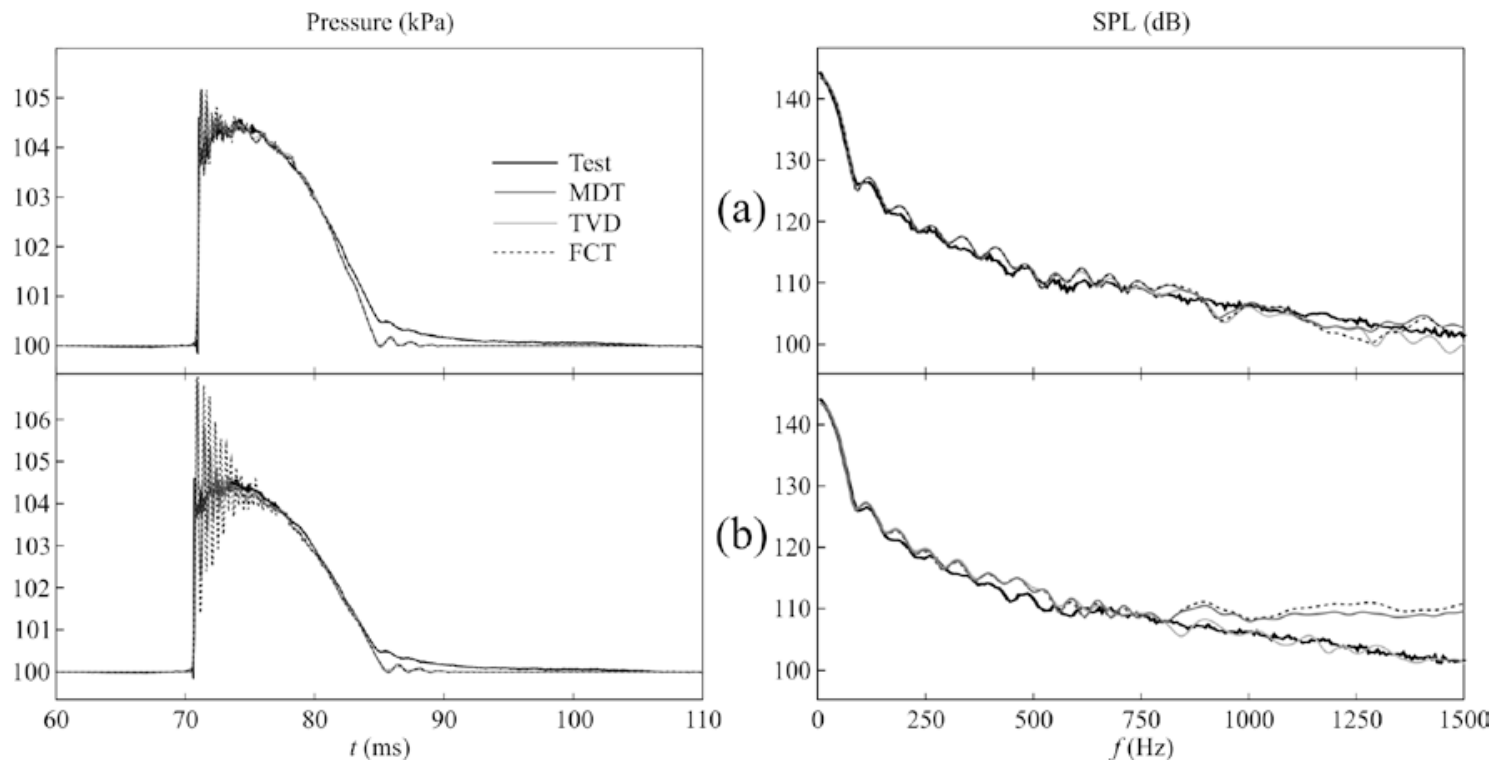

Figure 9: Pressure pulse as recorded at the second (a) and third (b) measurement stations, and results produced by the different methods.

In the frequency domain representation given in Fig. 9(a), one may first notice that the experimental pulse is now properly developed, as a relatively smooth and almost flat spectrum is observed for frequencies above $200 \mathrm{~Hz}$. Secondly, it is apparent that all the 
methods reproduce quite faithfully the experimental behaviour for frequencies below 500 Hz.

For frequencies above $500 \mathrm{~Hz}$ the results are more erratic, but in general the FCT and MDT methods predict a sound pressure level higher than that predicted by the TVD method. It is likely that this behaviour is related with the non-physical oscillations retained by those methods. This would also be confirmed by the fact that this tendency is especially clear at the highest frequencies represented. In any case, it appears that none of the methods produces any severe suppression of the medium-to-high frequency content of the signal, contrary to the results shown in [33], which seem to point out that all nonlinear schemes should suffer from large dissipation in combination with the generation of spurious oscillations throughout the entire wavenumber spectrum.

When considering the results obtained at the position of transducer 3, shown in Fig 9(b), all these effects are even more noticeable. As a result of the additional propagation, the residual oscillations previously shown by the MDT and FCT methods in the vicinity of the pulse ramp have grown dramatically, especially in the case of the FCT method, whereas the TVD results still follow quite closely the measured pressure trace. In the frequency domain a certain degradation of the quality of the results can be observed below $500 \mathrm{~Hz}$ for the three methods, and the agreement is somehow better than for transducer 2 between 500 and $750 \mathrm{~Hz}$. However, the main differences are found for frequencies above $800 \mathrm{~Hz}$ : it is here where the effect of the spurious oscillations in the MDT and FCT methods becomes apparent, with an almost linear increase of the sound pressure level with frequency that produces a significant deviation with respect to the measured spectrum. At the same time, the TVD method produces a very good approach to the measurements in these high frequencies. Even if such long propagation distances do not occur in engine exhaust systems, these results point to a certain superiority of the TVD method from a frequency-domain point of view.

\section{Application to a simple geometry}

Once the performance of the different methods was assessed in detail in one-dimensional cases as the shock-tube and the pulse propagation problems, the three-dimensional version of the method was applied to a simple but representative geometry, whose acoustic response cannot be properly accounted for by means of any one-dimensional model.

The geometry considered is shown in Fig. 10: it is a rectangular expansion chamber, with dimensions $129 \times 258 \times 344 \mathrm{~mm}^{3}$, meshed into cubes with $43 \mathrm{~mm}$ of side (for clarity, these are represented as spheres). The location of the inlet and outlet ducts was chosen so that a significant number of higher order modes should be excited. The transmission loss was obtained by simulating the improved impulse method proposed in [31], in which the chamber is excited by a pressure pulse similar to those shown in Fig. 9(a). In this way, there is significant content at all the relevant frequencies, and the transmission properties are directly obtained from the pulse transmitted by the chamber (as in section 5 , the length of the duct downstream of the chamber is chosen so as to avoid any overlaps with the pulse reflected at the open end). 


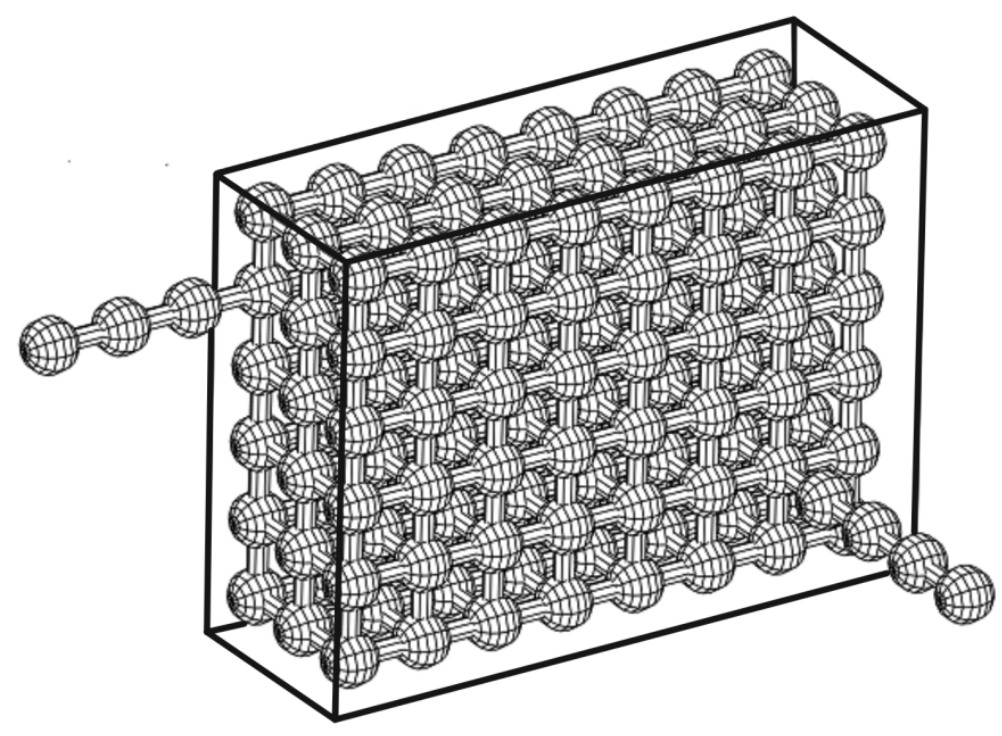

Figure 10: Geometry considered and mesh used.

The results are shown in Fig. 11, both for the transmitted pulses in the time domain and the resulting transmission loss in the frequency domain. In this last case, the transmission loss computed with the linear frequency-domain counterpart of the present method, which was described and validated in reference [34], is also included as a reference.
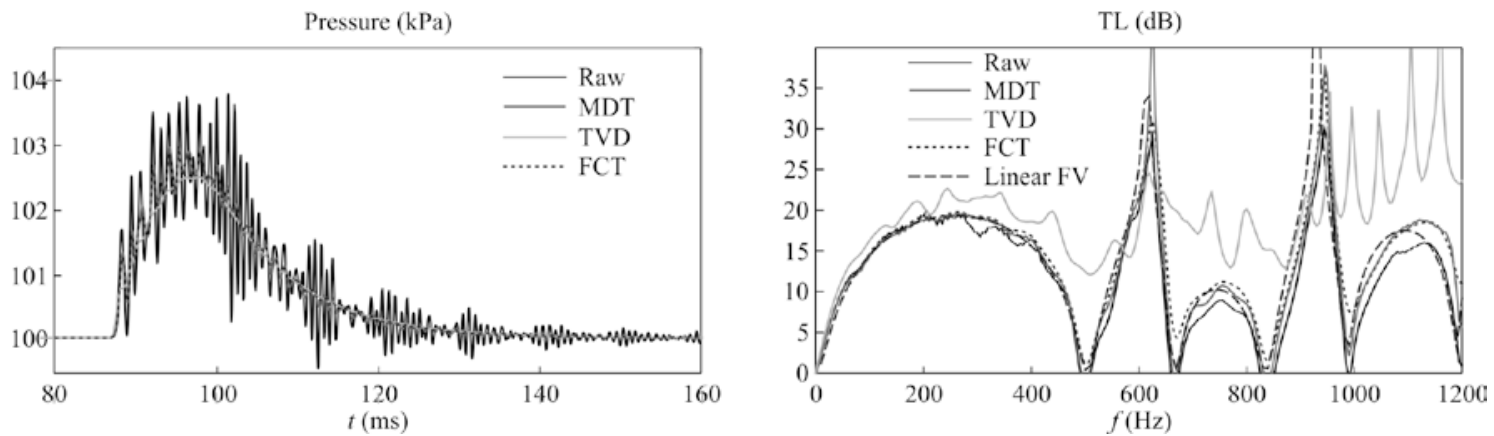

Figure 11: Pressure pulse transmitted by the chamber, and corresponding transmission loss, produced by the different flux limiters.

In the time domain representation, it can be observed that the results of the original method are severely affected by spurious oscillations that extend along the whole decay of the pulse. In all the other cases, such oscillations have been substantially removed. However, there are significant differences between the behaviour observed for the MDT and FCT methods, on one side, and for the TVD method on the other side. In the first case, some remaining oscillations persist, which are likely to be related with the actual wave dynamics inside the chamber, as multiple internal reflections occur, whereas in the case of the TVD method it appears that all the oscillations, both non-physical and possibly physical, have been suppressed, and thus some essential dynamics of the system may have been lost. 
This is confirmed by the transmission loss plots shown in Fig. 11, where it can be observed that the results obtained with the TVD method depart significantly from the behaviour predicted by the linear model, which is fully consistent with the geometry of the chamber and the position of the inlet and outlet ducts, whereas all the essential features present in the linear transmission loss have been reproduced by all the other methods, included the raw method without any flux limiter. In particular, the pass-bands observed are precisely related with the period of the oscillations retained by the FCT and the MDT methods, and obviously also present in the results of the original method, even if masked by the highfrequency non-physical oscillations, whose associated frequency is above the frequency range represented.

However, it is obvious from Fig. 11 that those non-physical oscillations have contaminated to some extent also the mid frequencies. This is particularly clear in the vicinity of the first three pass-bands, where instead of a zero value the original method predicts a negative transmission loss, i.e. some numerical noise is produced inside the chamber. At frequencies far from the pass-bands this may not be apparent, except for the fact that the original method gives everywhere, except at the resonant spikes, attenuation values lower than those corresponding to the linear solution, and thus this effect becomes visible only at the pass-bands themselves. This issue is also present in the results obtained with the MDT method, which might indicate that the expression of the momentum diffusion coefficient given by equation (12) is not the most convenient one for these particular flow conditions.

The results of the FCT method give a fair reproduction of the linear solution, without any negative values but with some clearly dissipative effects in some of the pass-bands and in the narrow-band resonant spikes, which are partially suppressed. Such dissipation is partly due to the use of the FCT technique, but it is also the expected behaviour when a pulse of relatively high amplitude (approximately 50 mbar in this case) is used as the excitation [31]. It can also be observed that the solution obtained starts to deviate quantitatively from the linear solution for frequencies above $1000 \mathrm{~Hz}$, which is consistent with the size of the mesh used.

The rather strange behaviour exhibited by the TVD results will now be examined in some detail, as the results obtained in section 5 suggested that the frequency-domain performance of this method compared favourably to the other ones in a one-dimensional case. The bad results obtained in the three dimensional case indicate that, as pointed out in section 3, the problem may lie in the treatment of end-volumes. While the solution adopted may give fair results when a mesh with a sufficiently large number of cells in each direction is used, this may not be the case for the relatively modest mesh used here. However, instead of increasing the number of volumes, what would penalize significantly the computational cost, a modification of the method aiming to reduce the effect observed was attempted.

The modification introduced consisted in changing the value of parameter $\xi$ in equation (28), whose suggested value is 0.5 . As this choice has a direct impact on the results of the method through equation (27), the effect of considering smaller $\xi$ values was studied. Such a change should reduce the influence of the additional TVD terms and consequently the effects of the ad hoc assumption at the end-volumes. 
The results obtained are shown in Fig. 12, again for both the transmitted pulses and the resulting transmission loss. It is apparent in the time domain representation that when reducing the value of $\xi$ the physically meaningful oscillations associated with the internal wave dynamics of the chamber are recovered. This indicates that the original value might have been optimal for a one-dimensional case, but too diffusive for this application. This is confirmed by the transmission loss results, in which it can be observed that the TVD predictions are closer to the linear solution the lower is the value of $\xi$ used. In fact, for $\xi=0.05$ the agreement with the linear solution is very good for frequencies below $1 \mathrm{kHz}$, the results being even better than those produced by the FCT method and shown in Fig. 11. For frequencies above $1 \mathrm{kHz}$, the results are almost indistinguishable from those obtained with the FCT or the MDT methods. It is likely that lower $\xi$ values would lead to negative transmission losses as those produced by the original and the MDT methods.
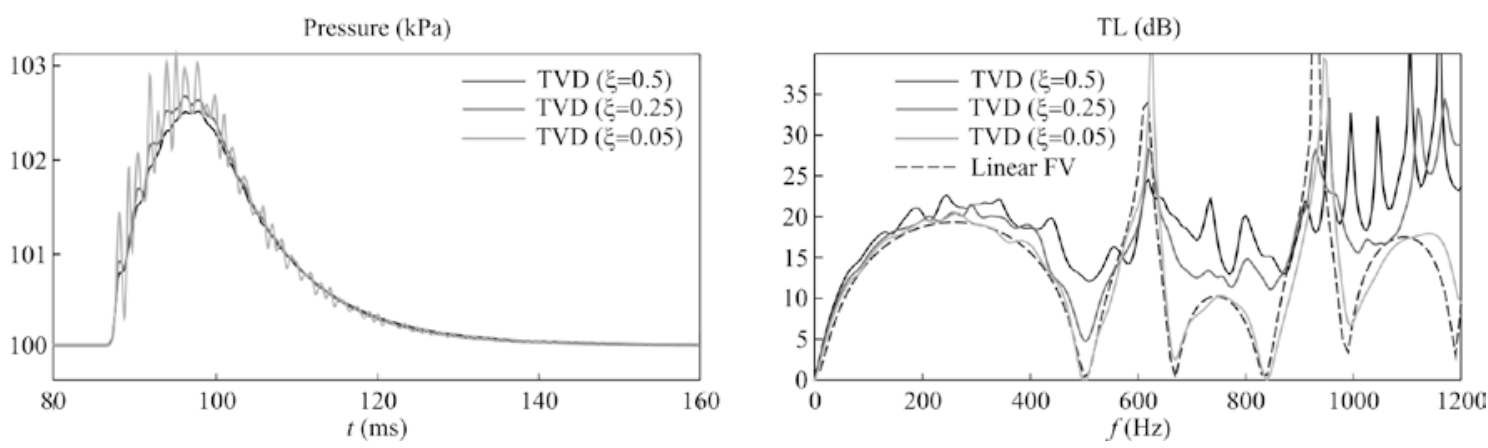

Figure 12: Effect of parameter $\xi$ in equation (28) on the pressure pulse transmitted by the chamber and the corresponding transmission loss obtained with the TVD method.

In any case, this analysis would not be complete if the influence of such a change in $\xi$ were not evaluated in the one-dimensional cases previously considered. The influence on the results of the shock tube problem can be observed in Fig. 13, where an expanded view of the most relevant part of the pressure and velocity results is shown. For clarity, only the two extreme values of $\xi$ have been considered.
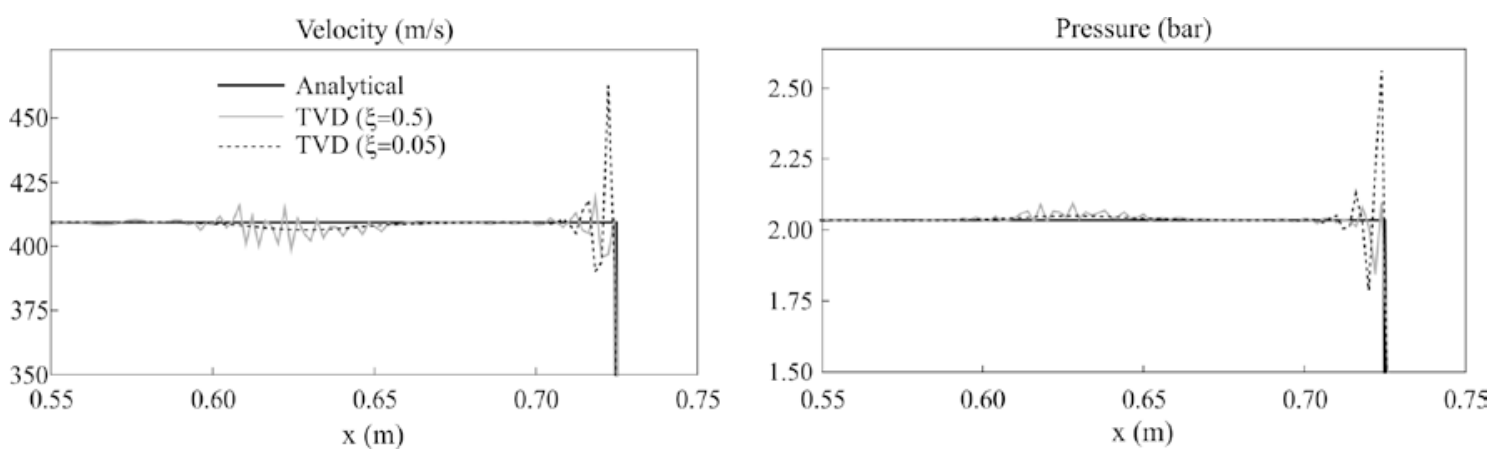

Figure 13: Effect of parameter $\xi$ in equation (28) on the pressure and velocity results of the shock-tube problem: detail at the contact discontinuity and the shock front.

It is clear that lowering the value of $\xi$ eliminates the spurious fluctuations previously noticed in the vicinity of the contact discontinuity, so that the results are now comparable to those obtained from the MDT and the FCT methods. However, this is achieved at the cost of a clearly insufficient removal of the spurious oscillations occurring at the shock 
wave front. Therefore, the use of such a small value for $\xi$ is not advisable in this particular problem.

The influence of $\xi$ on the TVD results for the pulse propagation problem can be checked in Fig. 14, where the pulses recorded after propagation along $20 \mathrm{~m}$ and their corresponding spectra are shown. It is apparent that the advantages previously exhibited by the TVD method regarding the absence of spurious oscillations and the good reproduction of the signal frequency content have been lost, the results being very similar to those provided by the MDT or the FCT methods.
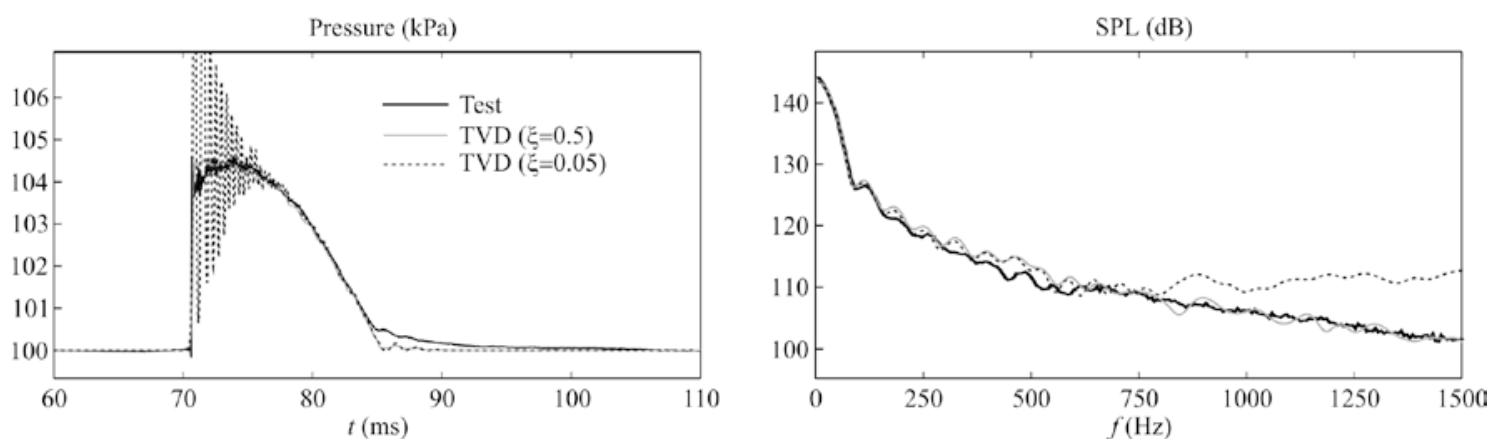

Figure 14: Effect of parameter $\xi$ in equation (28) on the pressure pulse as recorded at the third measurement station.

It appears thus that this new formulation of the TVD method is especially well suited for the analysis of the acoustics of cavities, but in any other of the conditions considered here its performance is clearly worse than that of the MDT and FCT methods.

\section{Summary and conclusions}

Staggered-mesh finite-volume models making use of a non-linear second order time and space discretization are affected by the occurrence of unphysical overshoots in the vicinity of discontinuities in the flow variables. In order to remove those overshoots, and as an alternative to the inclusion in the momentum equation of an equivalent friction force or a momentum diffusion term, two flux limiters commonly used in finite differences schemes have been considered: a Flux Corrected Transport (FCT) technique and a Total Variation Diminishing (TVD) method. In previous work by the authors [16], it was found that an FCT method with dissipation via damping together with the phoenical form of the antidiffusion term produced the best results when adapted to the staggered-mesh finitevolume model. In the case of the TVD methods, different formulations have been analyzed, and finally the Davis method was chosen for its adaptation in view of its relatively modest computational cost and its acceptable performance. Additionally, a momentum diffusion term (MDT) as described by Montenegro et al. [15] has been considered as a reference for evaluation purposes.

The resulting methods were first checked in the case of the well-known shock-tube problem. In this case, all the methods were comparably successful in removing the overshoots associated with the propagation of the shock wave. Additionally, they all exhibited a certain smoothing of the trailing side of the leftwards-moving rarefaction 
wave, and a considerable spreading of the contact discontinuity that in turn produces an overestimation of the pressure and an underestimation of the velocity at that point. In the case if the TVD method, these deviations were accompanied by spurious fluctuations, that did not get to disappear completely even when the mesh was improved in order to provide a better description of the contact discontinuity. Such fluctuations in the TVD solution were only removed when, at a later stage, the value of the $\xi$ parameter in equation (28) was reduced, but then the ability of the method to suppress the oscillations occurring at the shock wave front was severely penalized.

Secondly, in order to analyze the relative merits of the different schemes when describing the frequency domain behaviour of unsteady flows, they were applied to the simple but relevant case of a finite-amplitude pressure pulse propagating in a uniform duct, and the results obtained were compared with experimental measurements. In this case, it was the TVD method the one to provide the best approximation to the experimental results, both in the time and the frequency domains. In fact, none of the other methods considered is able to control the amplification over long distances of the unphysical oscillations produced following the rising ramp of the pulse, which could give rise to serious stability problems when interacting with any downstream boundary condition. Additionally, those oscillations lead to an excessively high content in the high frequencies. It was later checked that this applies also to the TVD scheme when value of the $\xi$ parameter is reduced.

Finally, the method was applied to a simple but representative geometry in which threedimensional features appear at relatively low frequencies. Initially, all the methods except the TVD were able to reproduce qualitatively the main features of the expected behaviour (characterized by the linear solution), but among the others only the FCT method was able to produce consistent transmission loss results without any negative value, i.e. without numerical noise generation inside the chamber. This might suggest that further analyses of the momentum diffusion coefficient used could produce an improvement in the results of the MDT method.

In the case of the TVD method, the initial results indicated a clear excess in dissipation that was attributed to the treatment of the end volumes, as this is the main issue in its implementation on a three-dimensional case. The consequent reduction of the value of the $\xi$ parameter produced a dramatic improvement in the results, which compare favourably with those obtained with any of the other methods. However, this modified formulation does not produce acceptable results in the one-dimensional problems studied, as commented above.

Therefore, it has not been possible to identify a single method that behaves properly in all the situations considered, with the possible exception of the FCT method. It is likely, however, that further work on the expression of the momentum diffusion coefficient might lead to acceptable results in all the cases also with the MDT method. The new formulation of the TVD method would be the solution of choice for cavity acoustics, but its use is not advisable in any other conditions.

\section{Acknowledgment}


M. Hernández is partially supported through contract FPI-S2-2015-1064 of Programa de Apoyo para la Investigación y Desarrollo (PAID) of Universitat Politècnica de València. 


\section{References}

[1]. D.E. Winterbone, R.J. Pearson, Design techniques for engine manifolds, Professional Engineering Pub. Ltd., 1999.

[2]. F. Payri, E. Reyes, J. Galindo, Analysis and modelling of the fluid-dynamic effects in branched exhaust junctions of I.C.E. J. Eng. Gas Turbines Power-Trans. ASME 123 (2001) 197-203.

[3]. A.J. Torregrosa, A. Broatch, L.M. García-Cuevas, M. Hernández, A study of the transient response of duct junctions: measurements and gas-dynamic modeling with a staggered mesh finite volume approach, Appl. Sci. 7 (2017) 480.

[4]. F. Payri, A.J. Torregrosa, M.D. Chust, Application of MacCormack schemes to I.C. engine exhaust noise prediction, J. Sound Vib. 195 (1996) 757-773.

[5]. A. Broatch, J.R. Serrano, F.J. Arnau, D. Moya, Time domain computation of muffler frequency response: comparison of different numerical schemes, J. Sound Vib. 305 (2007) 333-347.

[6]. G. Montenegro, A. Onorati, A coupled 1D-multiD nonlinear simulation of I.C. engine silencers with perforates and sound-absorbing material," SAE Int. J. Passeng. Cars Mech. Syst. 2 (2009) 482-494.

[7]. J. Galindo, A. Tiseira, P. Fajardo, R. Navarro, Coupling methodology of 1D finite difference and 3D finite volume CFD codes based on the Method of Characteristics. Mathematical and Computer Modelling 54 (2011) 1738-1746.

[8]. A. Onorati, M. Perotti, S. Rebay, Modelling one-dimensional unsteady flows in ducts: Symmetric finite difference schemes versus Galerkin discontinuous finite element methods. Int. J. Mech. Sci. 39 (1997) 1213-1236.

[9]. M. Perotti, Speed-up of a DFEM code for unsteady gas dynamics in pipes. Int. J. Mech. Sci. 41 (1999) 793-813.

[10]. G. Montenegro, A. Onorati, A. Della Torre, The prediction of silencer acoustical performances by 1D, 1D-3D and quasi-3D non-linear approaches, Computers \& Fluids 71 (2013): 208-223.

[11]. T. Morel, J. Silvestri, K. Goerg, R. Jebasinski, Modeling of engine exhaust acoustics, SAE Technical Paper 1999-01-1665, 1999.

[12]. S.M. Sapsford, VC.M. Richards, D.R. Amlee, T. Morel, M.T. Chappell, Exhaust system evaluation and design by non-linear modeling, SAE Technical Paper 920686, 1992.

[13]. G. Montenegro, A. Della Torre, A. Onorati, R. Fairbrother, D. Dolinar, Development and application of 3D generic cells to the acoustic modelling of exhaust systems, SAE Technical Paper 2011-01-1526, 2011.

[14]. T. Morel, R. Keribar, P.N. Blumberg, A new approach to integrating engine performance and component design analysis through simulation. SAE Technical Paper 880131, 1988.

[15]. G. Montenegro, A. Della Torre, A. Onorati, R. Fairbrother, Nonlinear quasi-3D approach for the modeling of mufflers with perforated elements and soundabsorbing material, Adv. Acoust. Vib. 2013 (2013) 546120.

[16]. A.J. Torregrosa, A. Broatch, F.J. Arnau, M. Hernández, A non-linear quasi-3D model with Flux-Corrected-Transport for engine gas-exchange modelling, J. Comput. Appl. Math. 291 (2016) 103-111. 
[17]. R.J. Pearson, D.E. Winterbone, The simulation of gas dynamics in engine manifolds using non-linear symmetric difference schemes, Proc. Inst. Mech. Eng. Part C-J. Eng. Mech. Eng. Sci. 211 (1997) 601-616.

[18]. A. Harten, High resolution schemes for hyperbolic conservation laws. J. Comput. Phys. 49 (1983) 357-393.

[19]. M. Vandervoorde, J. Vierendeels, E. Dick, R. Sierens, A new total variation diminishing scheme for the calculation of one-dimensional flow in inlet and exhaust pipes of internal combustion engines. Proc. Inst. Mech. Eng. Part D-J. Automob. Eng. 212 (1998) 437-448.

[20]. S. Jerez, M. Uh, A new TVD flux-limiter method for solving nonlinear hyperbolic equations, J. Comput. Appl. Math. 234 (2010) 1395-1403.

[21]. H.-D. Kim, Y.-H. Kweon, T. Setoguchi, A study of the weak shock wave propagating through an engine exhaust silencer system, J. Sound Vib. 275 (2004) 893-915.

[22]. P.D. Lax, B. Wendroff, Difference schemes for hyperbolic equations with high order of accuracy, Commun. Pure Appl. Math. 17 (1964) 381-398.

[23]. J.P. Boris, D.L. Book, Flux-corrected transport, J. Comput. Phys. 135 (1997) 172-186.

[24]. T. Ikeda, T. Nakagawa, SHASTA-FCT algorithm for the Cauchy-problem of the continuity equation, Math. Comput. 33 (1979) 1157-1169.

[25]. P.K. Sweby, High resolution schemes using flux limiters for hyperbolic conservation laws, SIAM J. Numer. Anal. 21 (1984) 995-1011.

[26]. S.F. Davis, A simplified TVD finite difference scheme via artificial viscosity, SIAM J. Sci. Stat. Comput. 8 (1987) 1-18.

[27]. F.J. Arnau, Numerical methods for the one-dimensional modelling of the gas exchange process (in Spanish), Editorial Reverté S.A., 2009.

[28]. G.A. Sod, Survey of several finite-difference methods for systems of non-linear hyperbolic conservation laws, J. Comput. Phys. 27 (1978) 1-31.

[29]. R. Courant, K.O. Friedrichs, H. Lewy, Über die partiellen Differenzengleichungen der mathematischen Physik. Math. Ann. 100(1928) 32-74.

[30]. J. Galindo, J.R. Serrano, F.J. Arnau, P. Piqueras, High-frequency response of a calculation methodology for gas dynamics based on Independent Time Discretisation, Math. Comput. Model. 50, (2009) 812-822.

[31]. F. Payri, J.M. Desantes, A. Broatch, Modified impulse method for the measurement of the frequency response of acoustic filters to weakly nonlinear transient excitations, J. Acoust. Soc. Am. 107 (2000) 731-738.

[32]. J.M. Desantes, J.R. Serrano, F.J. Arnau, P. Piqueras, Derivation of the method of characteristics for the fluid dynamic solution of flow advection along porous wall channels, Appl. Math. Model. 36 (2012) 3134-3152.

[33]. D. Fauconnier, E. Dick, Spectral analysis of nonlinear finite difference discretizations, J. Comput. Appl. Math. 246 (2013) 113-121.

[34]. A.J. Torregrosa, A. Broatch, A. Gil, D. Moreno, Analysis of acoustic networks including cavities by means of a linear finite volume method, J. Sound Vib. 331 (2012): 45754586. 